\title{
Experimental Verification of Four Wave Mixing in Lumped Optical Transmission Systems that Employ Mid-Link Optical Phase Conjugation
}

\author{
Mohammad A. Z. Al-Khateeb, Mary E. McCarthy, Andrew D. Ellis \\ Aston Institute of Photonic Technology, Aston University, Aston Triangle, Birmingham, UK \\ Authore-mail address: alkhamaz@aston.ac.uk
}

\begin{abstract}
We experimentally validate an analytical description of four wave mixing generated in lumped amplification systems that employ optical phase conjugation. The experimental results show good agreement with theoretical predictions within an error margin of $0.5 \mathrm{~dB}$.

OCIS codes: (190.4380) Nonlinear optics, four-wave mixing; (190.5040) Phase conjugation.
\end{abstract}

\section{Introduction}

Four wave mixing (FWM) effects in optical fibers limit the capacity of optical transmission systems. FWM has been theoretically described [1] for lumped optical transmission systems where it was used later to approximate nonlinear system performance [2]. Mid-link optical phase conjugation (OPC) [3] in system using lumped optical amplification can lead to significant compensation of inter/intra channel nonlinear mixing facilitating a higher system performance [4]. The level of nonlinearity compensation that can be achieved by mid-link OPC in lumped system is largely dependent on the level of power profile and dispersion symmetry in reference to the mid-link point.

In this paper, we experimentally validate, for the first time, an analytical closed form description of FWM in lumped optical transmission system that deploys mid-link OPC. The experimental results show an agreement with the theoretical predictions, and the fact that mid-link OPC in lumped optical transmission system (two spans, 100km each) can significantly reduce the nonlinear noise generated from FWM products within 5GHz bandwidth.

\section{Theory}

The power of FWM generated in a system is dependent on the result of the integration of the nonlinear Schrodinger equation (NLSE) over the distance of the link. In lumped optical amplified system, the FWM products power generated (at $\left.f_{F W M}=f_{i}+f_{j}-f_{k}\right)$ from the mixing of optical signals at frequency tones $\left(f_{i}, f_{j}\right.$, and $\left.f_{k}\right)$ can be written as:

$$
\begin{gathered}
P_{\mathrm{FWM}}\left(f_{i}, f_{j}, f_{k}\right)=P_{i} P_{j} P_{k}\left\{\frac{2 \mathrm{D} \gamma}{3\left(\alpha^{2}+\Delta \beta^{2}\right)} \frac{\sin (\mathrm{N} \Delta \beta \mathrm{L} / 4)}{\sin (\Delta \beta \mathrm{L} / 2)}\left[\alpha\left(e^{-\alpha L}+1\right) \sin \left(\frac{\Delta \beta \mathrm{L}}{2}\right)+\Delta \beta\left(e^{-\alpha L}-1\right) \cos \left(\frac{\Delta \beta \mathrm{L}}{2}\right)\right]\right\}^{2} \\
P_{F W M}\left(f_{i}, f_{j}, f_{k}\right)=P_{i} P_{j} P_{k}\left(\frac{\mathrm{D} \gamma}{3}\right)^{2} \frac{\sin (\mathrm{N} \Delta \beta \mathrm{L} / 2)^{2}}{\sin (\Delta \beta \mathrm{L} / 2)^{2}}\left[\frac{\left(1-\mathrm{e}^{-\alpha \mathrm{L}}\right)^{2}+4 \mathrm{e}^{-\alpha \mathrm{L}} \sin (\Delta \beta \mathrm{L} / 2)^{2}}{\alpha^{2}+\Delta \beta^{2}}\right]
\end{gathered}
$$

with [5] and without [1] mid-link OPC respectively, where $D$ is the degeneracy factor, $\gamma$ and $\alpha$ are respectively the nonlinear and attenuation coefficients of the fiber, $L$ is span length, $N$ is the number of spans in the system, and phase matching coefficient $\Delta \beta$ which is linearly dependent on the dispersion coefficient of the fiber $\left(\beta_{2}\right)$ is defined as: $\Delta \beta=-4 \pi^{2} \beta_{2}\left(f_{i}-f_{k}\right)\left(f_{j}-f_{k}\right)$. From Eqns. (1) and (2), it is clear that the FWM efficiency with mid-link OPC oscillates (in $\Delta \beta$ ) at half the rate of oscillation for the case without OPC, and vanishes as $\Delta \beta$ goes to zero when OPC is used.

\section{Experimental setup and results}

To verify the theory, we used two 100km standard single mode fiber (SMF) spans with lumped optical amplification, with and without mid-link OPC, as shown in Fig. 1. We measured the power of the FWM products, using a $150 \mathrm{MHz}$ resolution optical spectrum analyzer (OSA), as a function of frequency separation between two continuous wave $(\mathrm{CW})$ lasers. The two $\mathrm{CW}$ lasers were combined by a $3 \mathrm{~dB}$ coupler (entering the fiber with $5 \mathrm{dBm}$ power each, at around $1553 \mathrm{~nm}$ ). For the case of lumped system without mid-link OPC, only an erbium doped fiber amplifier (EDFA) is placed in between the two matching spans to compensate for the loss of the first span. For the case of lumped system with mid-link OPC, an OPC was installed by the end of the first span (pre-amplified to compensate for the insertion loss of the OPC). The OPC employed dual pump, polarization insensitive spectral inversion with pumps located at $1540.7 \mathrm{~nm}$ and $1570.1 \mathrm{~nm}$ at perpendicular polarizations which were combined using a polarization beam combiner (PBC). Fiber Bragg gratings (FBGs) were used to reduce the ASE noise generated 
from the high power EDFAs that amplified pumps. The two pumps (counter dithered at $60 \mathrm{MHz}$ and $600 \mathrm{MHz}$ to increase stimulated Brillouin scattering SBS threshold) and signal input to the OPC were combined using a 3dB coupler, then both $27 \mathrm{dBm}$ pumps along with signal input pass through a $100 \mathrm{~m}$ highly nonlinear fiber (HNLF) with a zero dispersion wavelength (ZDW) at $1557 \mathrm{~nm}$, dispersion slope of $0.024 \mathrm{ps} / \mathrm{nm}^{2} / \mathrm{km}$, nonlinear coefficient of $28 / \mathrm{W} / \mathrm{km}$. At the output of the OPC, the conjugated signals were filtered using optical band pass filter (BPF).

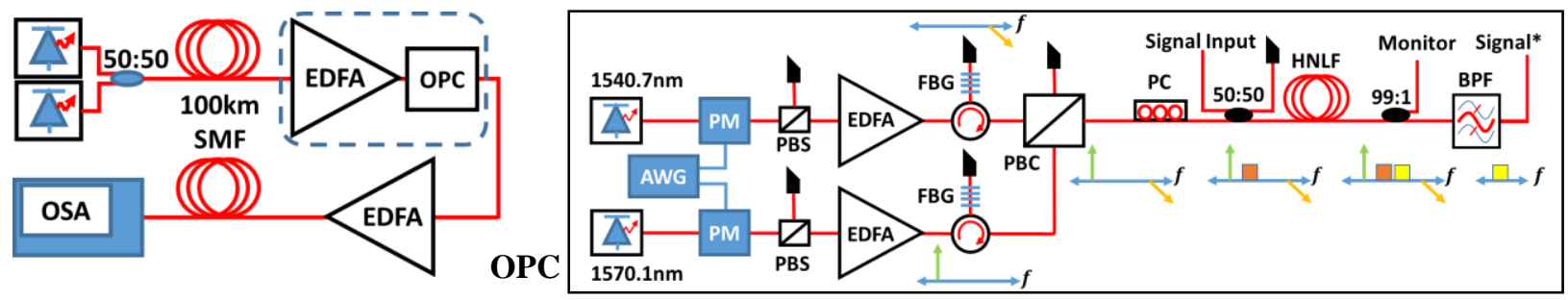

Fig. 1 Experimental setup: (left) two span lumped system with/without mid-link OPC, (right) dual pump polarization insensitive OPC.

Fig. 2 shows a comparison between theoretical, numerical simulation (of the previously described system) using VPI Transmission Maker 9.5, and experimental FWM product power as function of frequency separation. The experimental results show a good agreement with the theoretical and numerical predictions within a margin of error of less than $0.5 \mathrm{~dB}$ (at the peaks), the mismatching (at the FWM power nulls) between the experimental results and theoretical predictions is attributed to the limited OSA dynamic range and resolution and the CW lasers' linewidth. For the case where a mid-link OPC is installed, we attribute the error at low frequency separation to a slight mismatch in the pump dither, resulting in residual dithering components on the mixing product. For the case of a system with mid-link OPC the strongly phase matched nonlinear FWM product within the first fringe.
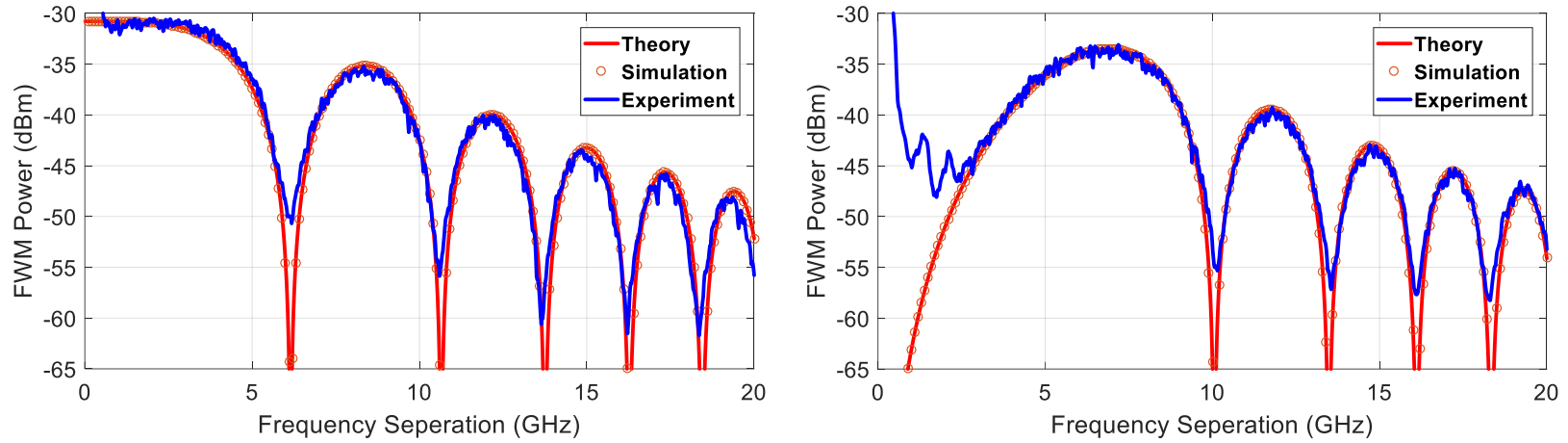

Fig. 2 FWM power as a function of frequency separation: (left) without mid-link OPC, (right) with mid-link OPC.

\section{Conclusion}

We experimentally verify the theoretical description of FWM for lumped optical transmission systems that deploy mid-link OPC. The experimental results show a good agreement with both theoretical and numerical predictions. Even in the presence of a long span length mid-link OPC can partially recover intra channel nonlinear effects corresponding to the first fringe of the four wave mixing efficiency characteristic.

Acknowledgment: this work was partially supported by the EPSRC via projects EP/J017582/1 and EP/L000091/1 (UNLOC and PEACE), Original data for this work is available through Aston Research Explorer (http://doi.org/10.17036/researchdata.aston.ac.uk.00000173)

\section{References}

[1] D. A. Cleland, A. D. Ellis, and C. H. F. Sturrock, "Precise modelling of four wave mixing products over $400 \mathrm{~km}$ of step-index fibre," Electron. Lett. 28, 1171 (1992).

[2] X. Chen and W. Shieh, "Closed-form expressions for nonlinear transmission performance of densely spaced coherent optical OFDM systems.," Opt. Express 18, 19039-19054 (2010).

[3] A. Yariv, D. Fekete, and D. M. Pepper, "Compensation for channel dispersion by nonlinear optical phase conjugation.," Opt. Lett. 4, 52 (1979)

[4] M. Al-Khateeb, M. Mccarthy, C. S. Costa, and A. D. Ellis, "Mid-Link Optical Phase Conjugation in Lumped Optical Transmission Systems," in Asia Communications and Photonics Conference 2016, OSA Technical Digest (Optical Society of America, 2016), paper AS2B.5.

[5] V. Pechenkin and I. J. Fair, "Analysis of Four-Wave Mixing Suppression in Fiber-Optic OFDM Transmission Systems With an Optical Phase Conjugation Module,” J. Opt. Commun. Netw., vol. 2, no. 9, p. 701, Aug. 2010. 